\title{
Atomic force microscopy for imaging human metaphase chromosomes
}

\author{
Tatsuo Ushiki* \& Osamu Hoshi \\ Division of Microscopic Anatomy and Bio-imaging, Niigata University Graduate School of Medical \\ and Dental Sciences, 1-757 Asahimachi-dori, Chuo-ku, Niigata, 951-8510, Japan; Tel: +81-25-2272058; \\ Fax:+81-25-2241767; E-mail: t-ushiki@med.niigata-u.ac.jp \\ * Correspondence
}

Key words: atomic force microscopy, chromatid, human, metaphase chromosome

\begin{abstract}
The present study introduces the principle of atomic force microscopy (AFM) and reviews our results of human metaphase chromosomes obtained by AFM. AFM imaging of the chromosomes revealed that the chromatid arm was not uniform in structure but had ridges and grooves along its length, which was most prominent in the late metaphase. The arrangement of these ridges and grooves was roughly symmetrical with the counterpart of the paired sister chromatids. AFM imaging of banded chromosomes also showed that the ridges and grooves were related to the G/Q-positive and G/Q-negative bands, respectively. At high magnification, the chromatid was seen to be produced by the compaction of highly twisted chromatin fiber loops, and its compaction tended to be stronger in the ridged regions of the chromosomes than in the grooved regions. Our AFM studies also showed the presence of catenation of chromatin fibers between the ridged portions of the chromatid in the late metaphase. Thus, AFM is useful for obtaining the three-dimensional surface topography not only in ambient conditions but also in physiological liquid conditions, and is expected to be an attractive tool for investigating the structure of chromosomes.
\end{abstract}

\section{Abbreviations}

AFM atomic force microscopy

TEM transmission electron microscopy

SEM scanning electron microscopy

SNOM scanning near-field optical microscopy

\section{Introduction}

The higher-order structure of chromosomes has been a subject of deep interest to researchers because it is intimately related to the mechanism of chromosome condensation and chromatid separation during mitosis. For this purpose, electron microscopy has been applied because chromosomes of micrometer size are difficult to analyze by light microscopy (see the review by Inaga et al. 2007). Thus, the ultrastructure of chromosomes has been studied by transmission electron microscopy (TEM) (e.g., Gall 1963, DuPraw 1970, Laemmli et al. 1978). Scanning electron microscopy (SEM) has also been applied to studies of chromosomes because it can provide threedimensional information on the sample surface (e.g., Pease \& Hayes 1966, Tanaka et al. 1970, Harrison et al. 1985, Sumner 1991, Inaga et al. 2002). Various models of the higher-order structure of chromosomes have been proposed by previous investigators who observed chromosomes by TEM and/or SEM. These include the folded-fiber model (DuPraw 1965), the successive helical coiling fiber model (Bak et al. 1977, Sedat \& Manuelidis 1978), the scaffold and radial loop model (Laemmli et al. 1978, Marsden \& Laemmli 1979), and the helical coiling of radial loops model (Rattner \& Lin 1985, 
Rattner 1992, Kireeva et al. 2004). However, the issue is still unresolved, probably owing partly to the difficulty of preserving the native chromosome structure during preparation, and also to the technical limitations for precise investigation of chromosomes by conventional TEM and SEM; whole chromosomes are too thick and condensed to be observed using TEM, while drying and metal-coating are required for SEM.

The atomic force microscope was invented in 1986 as an offspring of the scanning tunneling microscope (Binnig et al. 1986). This microscope has no lens but scans a sharp probing tip over a solid sample while monitoring the interaction force between the tip and sample surface. This creates three-dimensional information about the sample topography similar to that obtained by SEM. Thus, AFM has recently been applied to studies of the surface structure of various kinds of biological samples (see reviews by Engel et al. 1999, Ushiki 2003, Hörber \& Miles 2003, Ushiki \& Kawabata 2008). To our knowledge, AFM imaging of chromosomes is first reported in 1992 (de Grooth \& Putman 1992, Putman et al. 1992). Since these publications, a considerable number of AFM studies of chromosomes in various species have been published (e.g., Fritzsche et al. 1994, 1995, Tamayo \& Miles 2002, Thalhammer \& Heckl 2003). We have also been investigating the structure of human chromosomes by AFM and the results obtained have been published as several original articles and reviews (Ushiki et al. 1996, 2002, Hoshi \& Ushiki 2001, Kimura et al. 2004, Hoshi et al. 2004, 2006). In this review, we will first briefly introduce the principle and advantages of AFM, then discuss our AFM studies on the structure of human chromosomes, and finally provide some comments on the higher-order structure of chromosomes.

\section{Principle of atomic force microscopy (AFM)}

The basic concept of AFM is the measurement of forces between a probing tip and a sample surface (Meyer et al. 2004). For this purpose, a flexible cantilever with a sharp tip is most commonly used as the force sensor; when the tip approaches the sample surface, an interaction force occurs between the tip and the sample surface, which influences the position of the cantilever (Figure 1). In most instruments, the positional change of the cantilever, or cantilever deflection, is monitored by the beam-deflection method; a laser beam is reflected from the rear side of the cantilever and its deflection is monitored with a position-sensitive photodetector. Thus, the tipsample force can be accurately detected by the cantilever deflection. During AFM imaging, the sample is scanned by the tip in the $x y$-plane and the tipsample force is monitored and recorded. The signal is also used to keep the interaction force constant by electronic feedback to the scanner. A piezoelectronic $x y z$-scanner is usually used both to control the vertical position of the sample $(z)$ and to raster-scan the sample in the $x y$-plane.

Several modes of operation of AFM have been introduced according to the method of cantilever operation, but they can be divided generally into static and dynamic modes. In the static mode, the tip is simply dragged across the sample surface, and the tip-sample force is monitored by the static bending of the cantilever. Because the cantilever is permanently in contact with the sample surface during scanning, this mode is also often called the contact mode. In the dynamic mode, the sample surface is scanned with a vibrating cantilever, and the tipsample force is monitored by alteration of the resonance frequency or oscillation amplitude of the cantilever. The most commonly used dynamic mode measures a reduction of the oscillation amplitude as a feedback signal and is also referred to as the intermittent contact or tapping mode. The advantage of the dynamic mode is that it reduces the effect of lateral forces exerted on the cantilever during scanning. The vertical interaction force can also be controlled more finely in the dynamic mode $\left(10^{-9}\right.$ $10^{-10} \mathrm{~N}$ ) than in the contact mode (about $10^{-8} \mathrm{~N}$ ). In this regard, the dynamic mode is more commonly used for AFM imaging of chromosomes than the static mode, because the biological sample is very soft and easily deformed by the external force. We used the dynamic mode for AFM imaging of chromosome under ambient or liquid conditions.

AFM has several imaging modes (Putman et al. 1992). The AFM image produced by the feedback voltage to the $z$-scanner is called the 'constant force image' (or height image), which contains height information on the sample. On the other hand, the image can be obtained by displaying the change in cantilever deflection (in contact mode AFM) or 


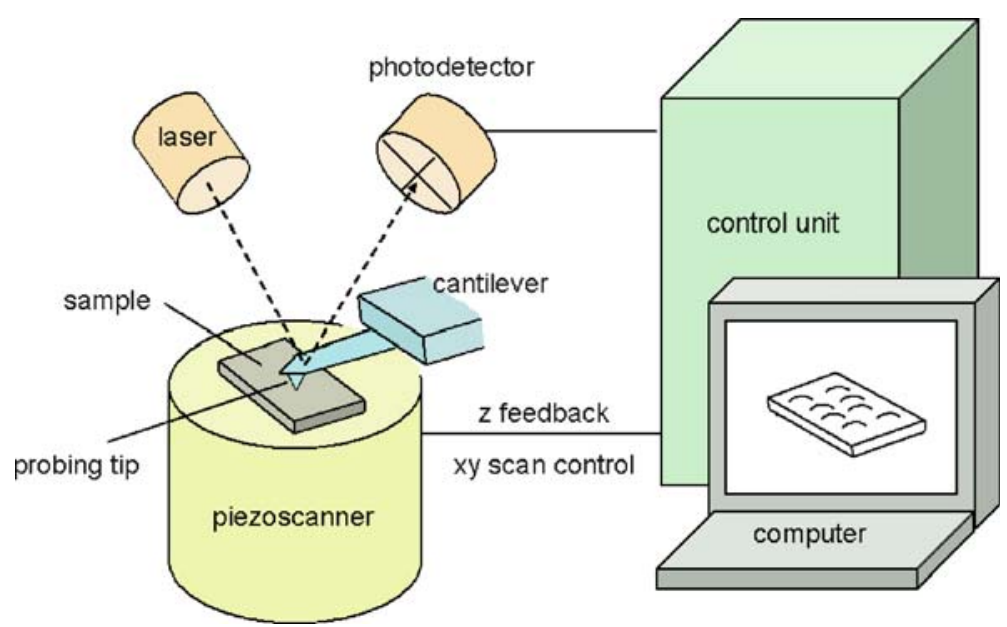

Figure 1. Schematic illustration of the atomic force microscope (AFM). The AFM has a sharp probing tip which scans over a sample while measuring the interaction force between the tip and the sample surface. The tip is attached to the end of a cantilever which serves as a force sensor. A piezoscanner is usually used both to control the vertical position of the sample $(z)$ and to raster-scan the sample in the $x y$-plane.

cantilever oscillation (in dynamic mode AFM); this is called the 'variable deflection image', which is especially useful for detection of the height change of the sample. In the studies described in this paper, we used the constant force image mode for showing height information of chromosomes.

\section{Advantage of AFM in the studies of chromosomes}

We previously attempted to compare the AFM images of the chromosomes with SEM images (Ushiki et al. 1996); after imaging by AFM, the specimens were coated with platinum-palladium in an ion coater and observed by SEM (Figures 2 and 3).

These observations clearly showed that the AFM images can compare with the SEM images. However, the results also suggested that there are several advantages of AFM imaging, which can be summarized as follows:

1. AFM can create a three-dimensional image of the chromosomes similarly to SEM.

2. With AFM, individual atoms have been observed in a few hard, crystalline samples. This means that the AFM has potential for providing threedimensional information on the surface topography of chromosomes at resolutions from the micrometer scale to the atomic scale.
3. Because SEM creates images by scanning the sample surface with an electron beam, samples need to be conductive-stained and/or metal-coated before SEM observation. This implies that precise information on the surface topography is hidden owing to the presence of the coating and/or staining substances. In contrast, AFM enables the direct observation of chromosomes without metal coating or any conductive treatment.

4. In contrast to a scanning electron microscope operated under vacuum condition, the atomic force microscope has the advantage of creating images not only in vacuum but also in air or liquid environments, indicating that one can expect to study the structure of wet chromosomes by AFM.

5. Usually, SEM images contain no quantitative information on the sample height. However, the atomic force microscope provides quantitative information in three dimensions, and the sample profile containing height information can be easily obtained from AFM images.

\section{Sample preparation}

Sample preparation methods are basically the same as those for TEM or SEM. Most commonly, 
chromosome spreads are used for observation of chromosomes by AFM, because the specific number of chromosome can easily be determined in the spread. Isolated chromosomes are also used for AFM observation under liquid conditions, in order to study the structure of wet and unfixed chromosomes. Sample preparations used in our studies are shown in flowchart form in Figure 3.

\section{Chromosome spreads}

Human lymphocytes collected from heparinized peripheral blood of healthy male donors were cultivated in karyotyping culture medium (PB-max, Gibco, BRL, UK) with $10 \%$ fetal calf serum for $72 \mathrm{~h}$ at $37^{\circ} \mathrm{C}$, under $5 \% \mathrm{CO}_{2}$ and $95 \%$ air. In some cases, lymphocytes after cultivation were arrested in metaphase by adding colcemid to the culture medium at a final concentration of $0.05 \mu \mathrm{g} / \mathrm{ml}$ for $1 \mathrm{~h}$. The cell suspension was then exposed to $75 \mathrm{mM} \mathrm{KCl}$ for $30 \mathrm{~min}$ at room temperature and fixed with Carnoy's solution (methanol-acetic acid 3:1 v/v). Chromosome spreads were then formed by dropping the cell suspension onto glass slides, and leaving them in a humid atmosphere.

\section{Isolated chromosomes}

Cells from the human cell line BALL-1 (RCB0256), obtained from RIKEN Cell Bank (Riken, Tsukuba, Japan), were grown in a $40 \mathrm{ml}$ flask in culture medium (RPMI 1640, Invitrogen Japan, Tokyo) at $37^{\circ} \mathrm{C}$ under an atmosphere containing $5 \% \mathrm{CO}_{2}$ and $95 \%$ air. After arrest with $0.06 \mu \mathrm{g} / \mathrm{ml}$ colcemid for $12 \mathrm{~h}$, the cells were suspended and centrifuged at $190 \mathrm{~g}$ for $10 \mathrm{~min}$ at $4^{\circ} \mathrm{C}$, resuspended as a pellet in $10 \mathrm{ml}$ of the culture medium for $20 \mathrm{~min}$, centrifuged again at $190 \mathrm{~g}$ for $10 \mathrm{~min}$, and then exposed to $75 \mathrm{mM} \mathrm{KCl}$ for $15 \mathrm{~min}$. These cells were collected by centrifugation at $190 \mathrm{~g}$ and isolated using the hexylene glycol method (Wray \& Stubblefield 1970, Hoshi et al. 2006).
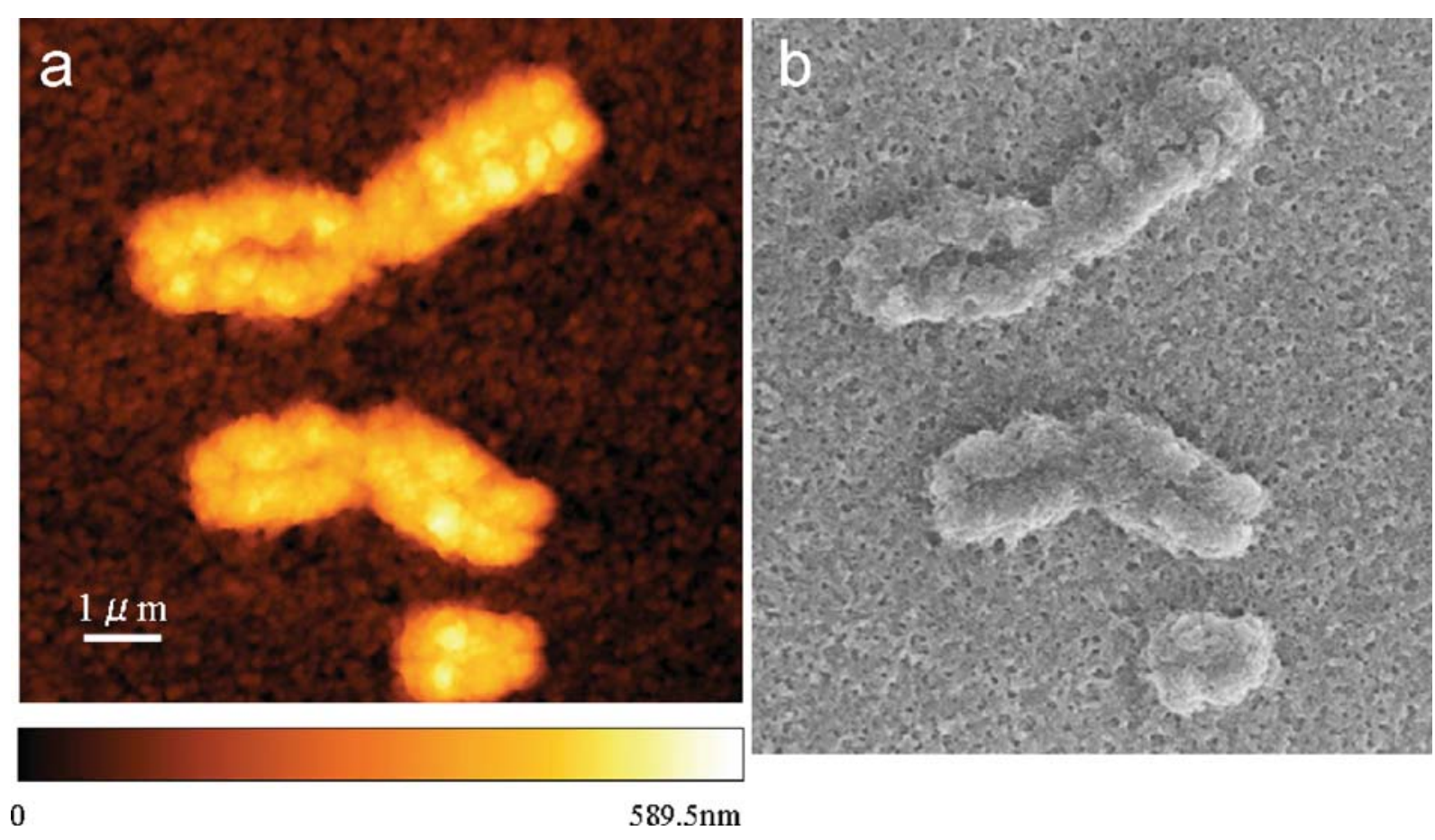

Figure 2. Comparison of the AFM image (a) with the SEM image (b) of human metaphase chromosomes. After imaging by AFM, the specimen was coated with platinum-palladium in an ion coater and observed by SEM (see also Figure 3). The height of the specimens in the AFM image is displayed as color gradations. The AFM image corresponds well to the SEM image, but has the advantage in giving height information of non-coated samples. (Reproduced with permission from Ushiki et al. 1996.) 


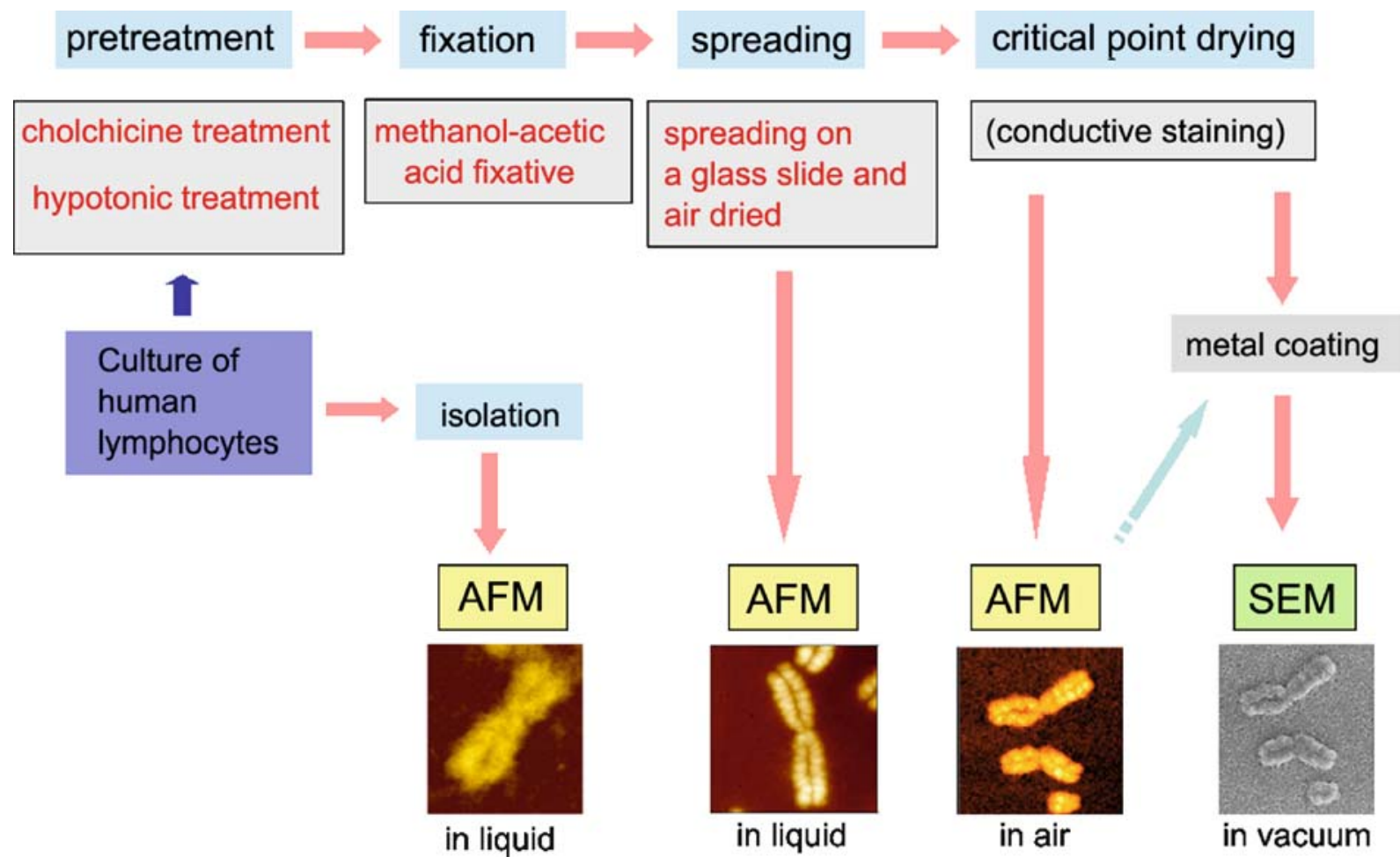

Figure 3. Flowchart showing the basic methods of chromosome sample preparation for AFM imaging.

\section{Human metaphase chromosomes observed by AFM}

\section{AFM of dried chromosomes}

The simplest technique is to observe dried chromosomes under ambient conditions by AFM. The contours of metaphase chromosomes with a centromere and paired chromatids are clearly observed by AFM. However, each chromatid of the air-dried chromosomes is flattened, usually about $1 \mu \mathrm{m}$ in width and about 40-50 $\mathrm{nm}$ in height (Hoshi \& Ushiki 2001, Hoshi et al. 2004). The chromosome arms were rather uniform in structure with a flat surface, and, even at high magnification, they showed only a granular appearance. This is probably due to the deformation artifact caused by surface tension during air-drying, as well as the presence of a blanket-like layer of cytoplasmic debris draped over the chromosome during the standard method for preparation of the chromosome spread.

To minimize the deformation artifacts caused by air-drying, we have applied the critical-point drying method for sample preparation of chromosomes; the same technique is commonly used in SEM (Ushiki et al. 2002). Briefly, the chromosome spreads produced by dropping the cell suspension onto the glass slide were dried for a few minutes, and hydrated again by immersion in phosphate buffer for $30 \mathrm{~min}$ or more. They were then treated with a $1 \%$ tannic acid solution for $15 \mathrm{~min}$, washed in the buffer for $15 \mathrm{~min}$ (3 changes, $5 \mathrm{~min}$ each), and treated with a $1 \% \mathrm{OsO}_{4}$ solution; this treatment is useful not only for hardening chromosomes but also for preventing the shrinkage of the samples during preparation. These samples were dehydrated in a graded series of ethanol, and critical-point dried in $\mathrm{CO}_{2}$.

To remove the cytoplasmic debris, some investigators recommend an enzymatic digestion with pepsin and/or RNase for AFM observation of chromosomes (Tamayo et al. 1999, Tamayo \& Miles 2002). In our case, we carefully selected chromosome spreads with little cytoplasmic debris by phasecontrast light microscopy and used these samples for AFM imaging. 
In these specimens with appropriate treatment, human chromosomes were measured to be about 200-350 nm in height, and often showed circumstantial grooves and ridges on the surface of the chromatid arms (Ushiki et al. 2002) (Figure 4). The arrangement of the grooves and ridges was roughly symmetrical with that of the counterpart paired sister chromatid. At high magnification, the surface of the chromosome often showed a globular appearance, but careful studies suggested that they were produced by strongly twisted fibrous structures about $50 \mathrm{~nm}$ thick (Ushiki et al. 2002, Hoshi et al. 2007b). The degree of twisting of fibrous structures (or chromatin fibers) was marked in the ridged portions, while it was somewhat weaker in the grooved portions.
Although not commonly found, loops of chromatin fibers were recognized at the periphery of metaphase chromosomes that were accidentally loosened during the sample preparation (Figure 5). This suggests that chromatin fibers are anchored in places along the axis of the chromatid to form a number of loops, which are strongly twisted at (and below) the chromatid surface.

AFM of the chromosomes further revealed that a pair of sister chromatids was not completely separated even in late metaphase, but was connected by fibrous structures about 50-60 nm thick, suggesting the presence of catenation of chromatin fibers between the paired chromatids (Ushiki et al. 2002). While the catenation was present throughout the gap

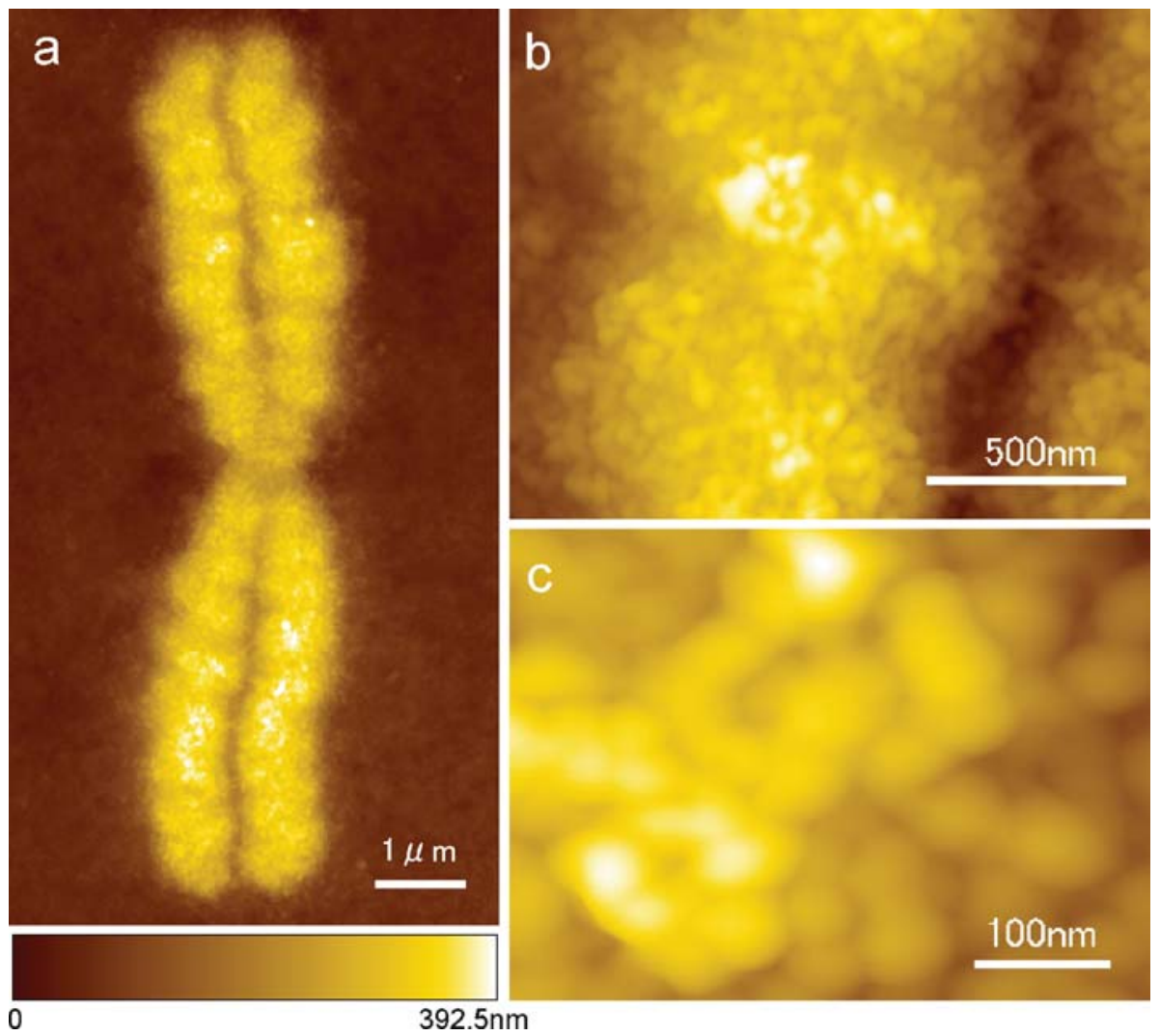

Figure 4. AFM images of a human metaphase chromosome 1. Chromosome spreads on a glass slide were immersed in a buffer, treated with a $1 \%$ tannic acid solution and $1 \% \mathrm{OsO}_{4}$, critical-point dried in liquid $\mathrm{CO}_{2}$ and observed in ambient conditions by dynamic mode AFM. (a) The overall appearance of the chromosome. Ridges and grooves are observed in the sister chromatids. The position of ridges and grooves is the same in each of a pair of sister chromatids. The height scale is shown by the gradation bar. (b) A closer view of a part of the chromatid arm. Both ridges and grooves of the chromatid are apparently composed of aggregations of globular or fibrous structures. The aggregations are stronger in the ridges of the chromatid than in the grooves. (c) Higher magnification of a part of the chromatid arm. Strongly twisted chromatin fibers of about 50-60 nm are observed at the surface. (For AFM imaging, commercially available rectangular silicon cantilevers with a nominal spring constant of $42 \mathrm{~N} / \mathrm{m}$ were used for AFM imaging in air; the resonance frequency was normally $300 \mathrm{kHz}$ in air.) 


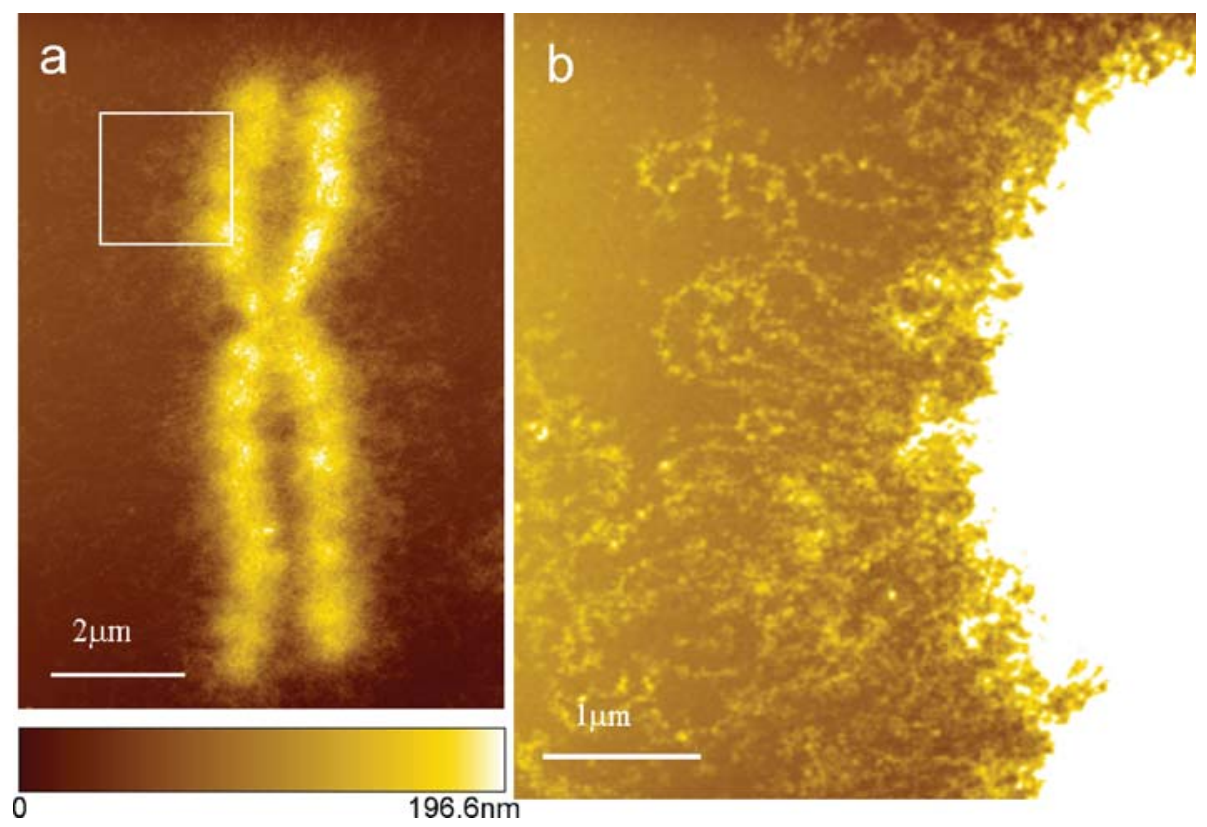

Figure 5. AFM images of a human chromosome prepared in the same way as described in Figure 4. (a) The overall appearance of the chromosome, in which chromatin fibers were accidentally unraveled during the sample preparation. The height scale is shown by the gradation bar. (b) Closer view of a part of the chromosome arm indicated by the white square in (a). Loops of chromatin fibers are clearly recognized at the periphery of this chromosome.

between the paired chromatids in prometaphase, it was restricted to the ridged portions of the chromatids in late metaphase, especially in mitosis without colchicine treatment.

\section{AFM of wet chromosomes}

In order to analyze chromosomes under conditions much closer to the physiological state, human chromosomes were also observed in a liquid environment by AFM (Hoshi et al. 2004, 2006). Chromosome spreads of human lymphocytes were briefly dried in air in order to fix them onto the glass slides, and were then immersed in phosphatebuffered saline. The height of the chromosomes was generally about 200-300 nm but sometimes ranged up to $600 \mathrm{~nm}$ (Hoshi et al. 2004), probably depending on timing of drying and rehydration during preparation.

The surface of chromatids in the wet chromosomes was characterized by the presence of alternating ridges and grooves, the arrangement of which was roughly symmetrical with the counterpart paired sister chromatids (Figure 6). These features were almost the same as those found in the critical-point dried chromosomes, but the patterns of ridges and grooves were more apparent in the wet chromosomes than in the critical-point dried chromosomes. It should be noted that the number and arrangement of the ridges and grooves appeared to be specific to the type of chromosome; for example, the number of ridges was usually 17 for chromosome 1 , and 16 for chromosome 2 in late metaphase (Hoshi et al. 2004). Some ridges were connected with those of the counterpart paired chromatid and they closely associated with each other.

For study of the structure of unfixed (or native) chromosomes in liquid environment, isolated human metaphase chromosomes were also observed by AFM (Hoshi et al. 2006). Isolated chromosomes as described above were adsorbed onto a silane-coated glass slide by dropping the chromosome suspension onto the slide. The chromosomes to be studied were selected in the hexylene glycol buffer with a phasecontrast microscope, and were observed by AFM in the same buffer solution (Figure 7). These chromosomes were about $1 \mu \mathrm{m}$ in width and ranged from 400 to $800 \mathrm{~nm}$ in height. At high magnification, globular or fibrous structures about $30-50 \mathrm{~nm}$ thick were observed on the surface of each chromatid, 


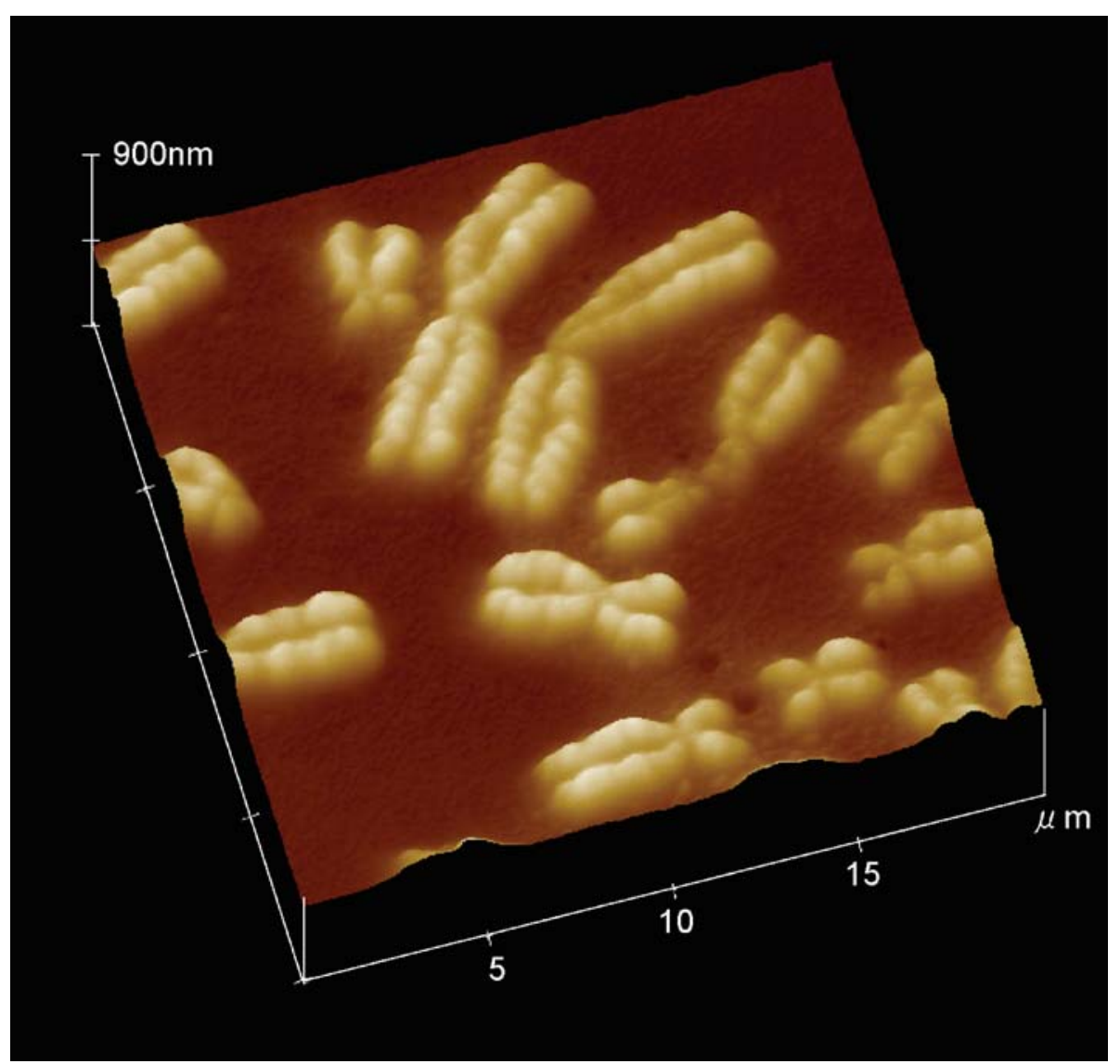

Figure 6. AFM image of fixed chromosomes under liquid conditions. Spreads of human metaphase chromosomes were immersed in phosphate-buffered saline and observed in the same solution with by dynamic mode AFM. Ridges and grooves are prominent in the sister chromatids of the chromosomes. ( $\mathrm{V}$-shaped silicon nitride cantilevers with oxide-sharpened tips with nominal spring constant of $0.37 \mathrm{~N} / \mathrm{m}$ were used for imaging in liquid; the resonance frequency was normally about $12.5 \mathrm{kHz}$ in liquid.)

which corresponded well to the findings obtained in the critical-point dried samples. The partial connection of paired sister chromatids with entangled chromatin fibers was also observed in these chromosomes.

\section{Chromosome bands observed by AFM}

The presence of chromosome banding was first introduced by Caspersson et al. (1970), who discovered that quinacrine mustard produces consistent fluorescent banding patterns along each human chromosome in the chromosome spread. Since then, a number of chromosome banding techniques have been reported by various investigators (see the review by Bickmore \& Craig 1997). The most common methods of chromosome banding are for
C- (centromere), Q- (quinacrine), G- (Giemsa) and R- (reverse) banding.

There have been various reports on the ultrastructure of banded chromosomes by TEM and/or SEM (Schwarzacher et al. 1975, Bath 1976, Harrison et al. 1981, 1983). In AFM studies of chromosomes, several investigators including us have also been interested in the structure of banded chromosomes (Musio et al. 1997, Sahin et al. 2000, Hoshi \& Ushiki 2001, Thalhammer et al. 2001, Fukushi \& Ushiki 2005). Among them, AFM studies on G-banding clearly showed that the air-dried G-banded chromosomes had clear ridges and grooves corresponding to the G-positive and G-negative bands, respectively (Hoshi \& Ushiki 2001) (Figure 8). AFM also revealed that G-banded chromosomes were formed by aggregation of chromatin fibers about $50-100 \mathrm{~nm}$ 

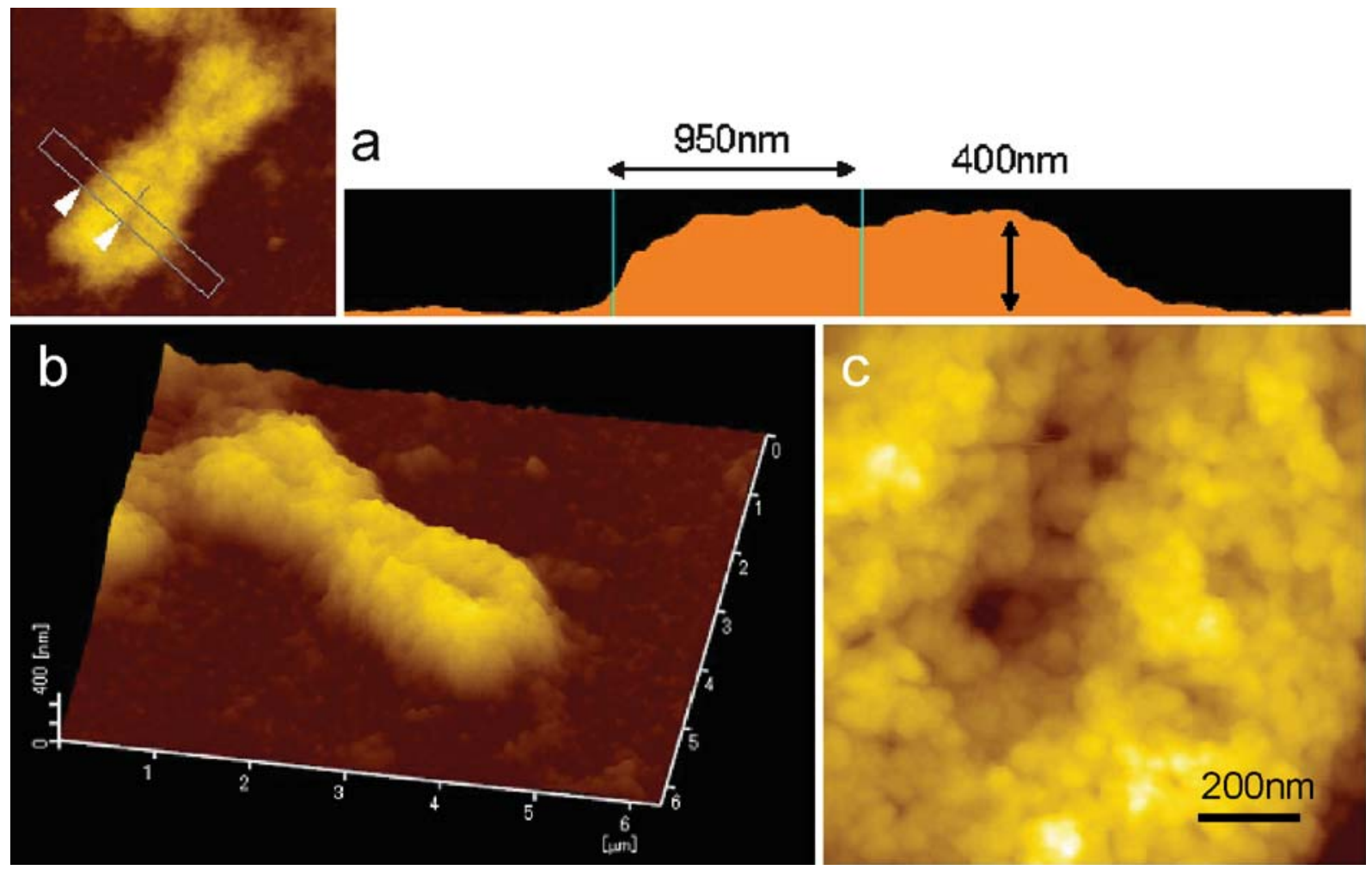

Figure 7. AFM images of unfixed chromosomes under liquid conditions. Chromosomes of the human cell line BALL-1 were isolated by the hexylene glycol method, mounted on a glass slide, and observed in a hexylene glycol buffer solution by dynamic mode AFM. The operation conditions are almost the same as in Figure 6. (a) The profile (right) is produced by calculating the average of the height in the region indicated by the box in the micrograph at left. The width of the chromatid, indicated by arrowheads, is $950 \mathrm{~nm}$, while the height of the chromatids is about $400 \mathrm{~nm}$ in this specimen. (b) Three-dimensional representation of the chromosome. (c) Closer view of a part of the chromatid showing an aggregation of globular or fibrous structures. (Reproduced with permission from Hoshi et al. 2006.)

in diameter, and the arrangement of these fibers differed between the G-positive and G-negative regions; G-positive ridges were composed of an aggregation of chromatin fibers, while the fibers were sparse and loose in G-negative grooves. It is interesting that the grooves were very shallow, or sometimes unclear in the G-negative portions of critical-point dried chromosomes. However, at higher magnification, the chromatin fibers were looser in the G-positive portions of the critical-point dried chromosomes, suggesting that certain proteins were extracted dominantly from G-negative portions by trypsin treatment. This probably caused the collapse of G-negative portions due to the surface tension of water during air-drying (Hoshi \& Ushiki 2001).

AFM of Q-banded chromosomes also revealed that the structure of the Q-banded chromosomes is essentially the same as that of the G-banded chromosomes, in that the Q-positive and Q-negative portions correspond roughly to the ridges and grooves of the Q-banded chromosomes (Ushiki et al. 2002) (Figure 9). AFM studies further showed that the ridges and grooves of the Q-banded chromosomes are the same as the ridges and grooves of non-banded chromosomes as described above. Chromatin fibers bridging the gap between paired chromatids were also prominent in the ridged portions of the banded chromosomes.

\section{The structure of human metaphase chromosomes}

The structure of human metaphase chromosomes based on our AFM studies can be summarized as follows:

1. The chromatid arm of metaphase chromosomes is not uniform in structure but has ridges and 

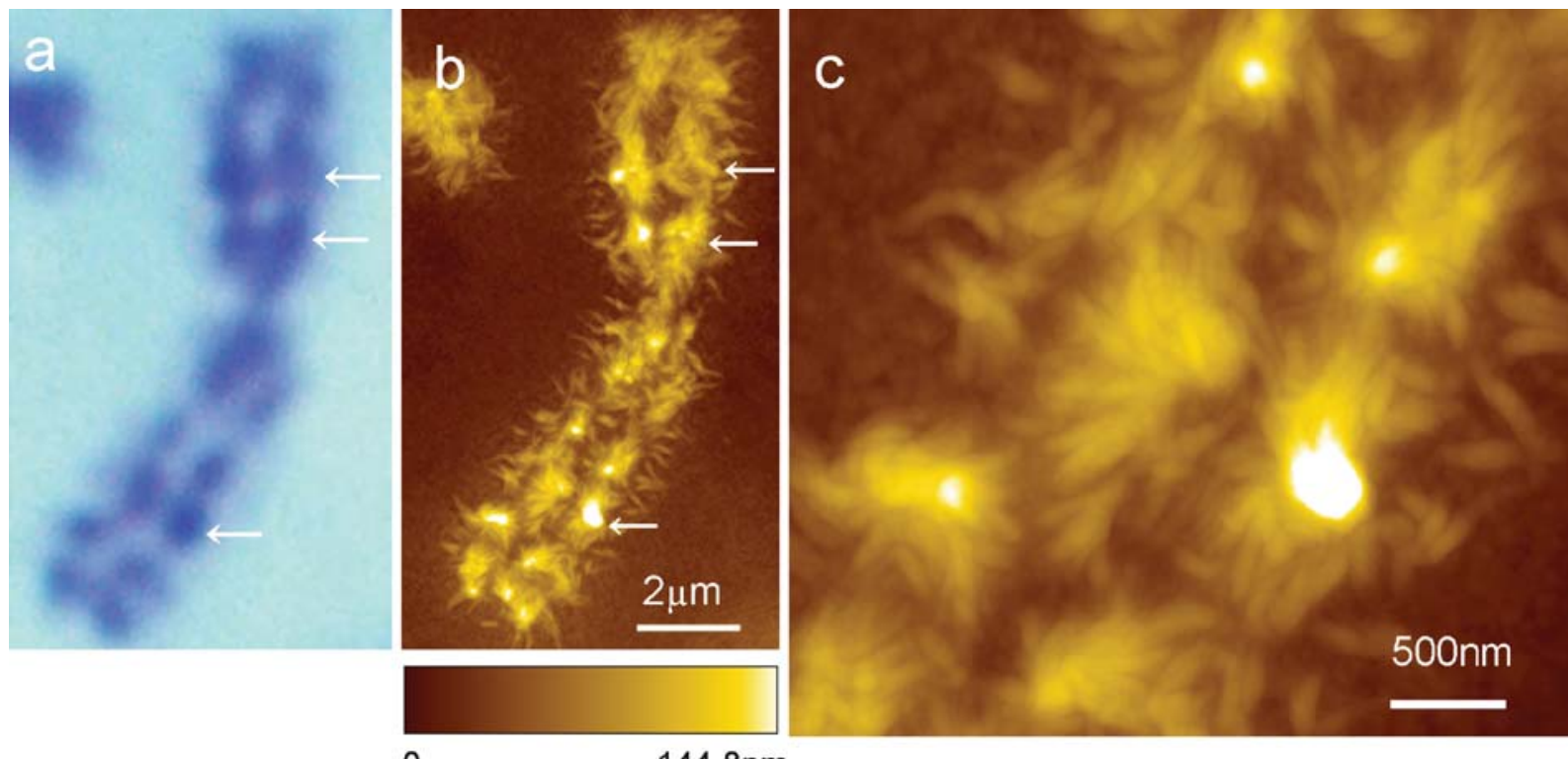

0

$144.8 \mathrm{~nm}$

Figure 8. Light-microscopic (a) and AFM (b, c) images of a G-banded chromosome 1, which was simply air-dried after G-banding with $0.025 \%$ trypsin digestion. (a, b) Comparison between (a) and (b) clearly shows that G-positive (dark) and negative (pale) bands correspond to ridges and grooves, respectively. The height scale is shown as a gradation bar. (c) Closer view of a part of (b). G-positive ridges are composed of densely packed chromatin fibers, while chromatin fibers are rather sparse in the G-negative grooves. (Reproduced with permission from Hoshi \& Ushiki 2001.)

grooves along its length, which are most prominent in late metaphase. The arrangement of these ridges and grooves is roughly symmetrical with the counterpart paired sister chromatid, and the number and arrangement of the ridges and grooves are considered to be specific to the type of chromosome. The ridges and grooves roughly correspond to the G/Q-positive and G/Q-negative bands, respectively.

2. The chromatid is filled with granular and/or fibrous structures about $50-60 \mathrm{~nm}$ in diameter on its surface. The appearance of chromatin fiber loops at the periphery of chromosomes suggests that the chromatid is produced by the compaction of highly twisted chromatin fiber loops in each portion.

3. The compaction of the chromatin fibers tended to be stronger in the ridged regions of the chromosomes than in the grooved regions.

4. The paired chromatids are usually connected to each other by chromatin fibers. These connections are especially apparent in the ridged regions of the chromatids of the late metaphase chromosomes.

A schematic representation of the structure of human metaphase chromosomes based on our AFM findings is shown in Figure 10. We consider that the structure of the chromosome arm is not uniform but heterogeneous because of the presence of highly condensed and less condensed regions in each chromosome; the highly condensed regions are observed as ridged portions of the chromatid, which are also related to G/Q-positive bands. The less condensed regions, on the other hand, are observed as grooved portions, which are related to G/Q-negative bands. Previous biochemical studies showed that the DNA of G-positive bands is AT-rich compared with the GC-rich content of G-negative bands (Holmquist et al. 1982). Thus, it is probable that the ridged portions with AT-rich DNA are composed of relatively highly condensed heterochromatin, which is also highly condensed in the metaphase chromosomes.

The presence of alternating ridges and grooves in metaphase chromosomes might be considered in connection with the spiral arrangement of the sister chromatid in the chromosomes of various species (Makino 1936, White 1940). Ohnuki (1968) treated human lymphocytes with a special hypotonic solution and showed that the sister chromatids of the human metaphase chromosome were composed of a spiral or zigzag fiber. Our AFM observations of these 


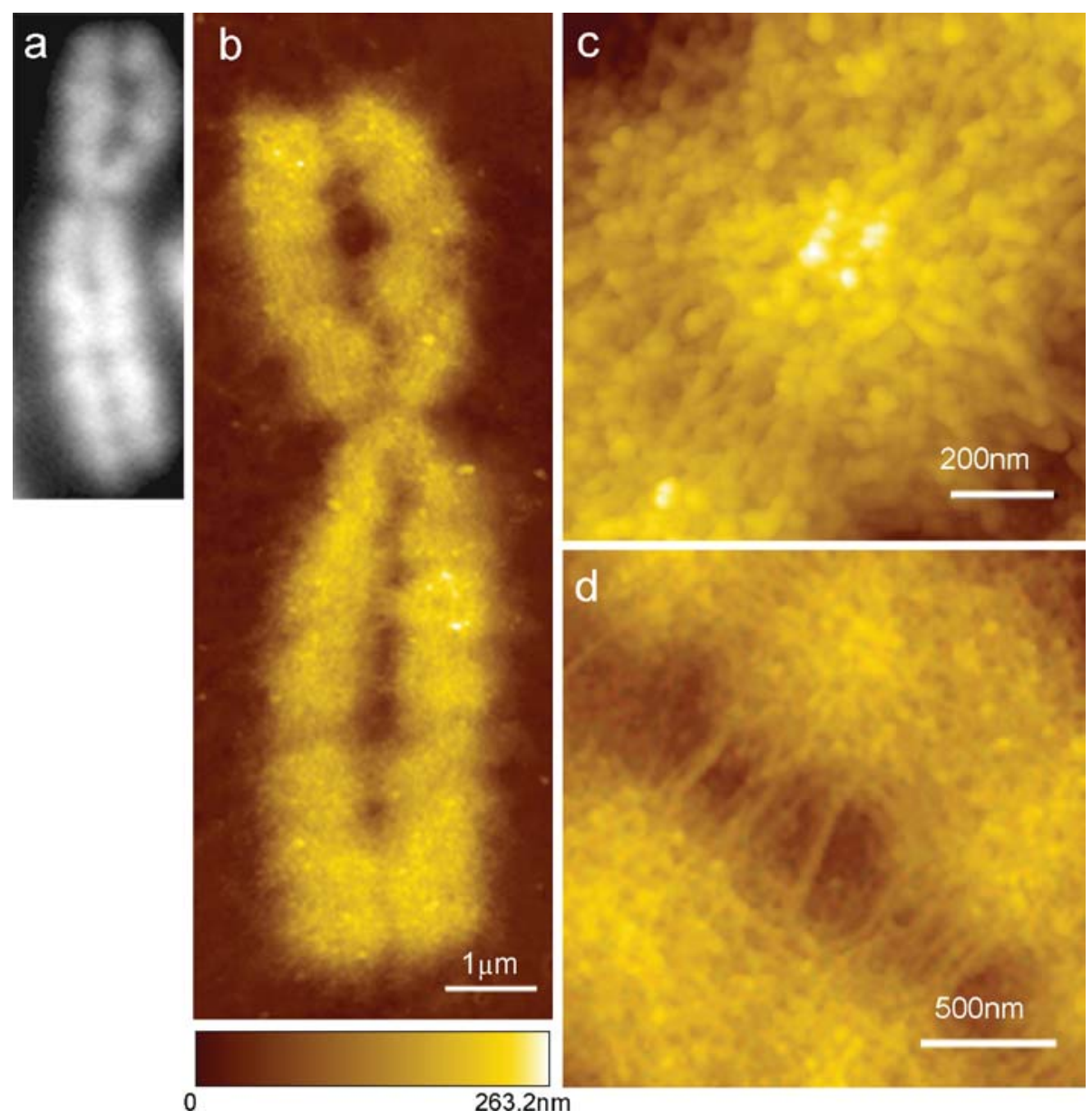

Figure 9. Light-microscopic (a) and AFM (b-d) images of a Q-banded chromosome 2. For AFM, the chromosome was stained with quinacrine mustard, treated with a $1 \%$ tannic acid solution and $1 \% \mathrm{OsO}_{4}$, and critical-point dried in liquid $\mathrm{CO}_{2}$. (b) The overall appearance of the Q-banded chromosome. The ridges and grooves are distinct in the chromosome arms. The height scale is shown as a gradation bar. (c) Closer view of a part of the chromatid. Chromatin fibers are strongly twisted and densely packed in the Q-positive portion, while they tend to be loose and run in a longitudinal direction in the Q-negative portion. (d) This micrograph shows fibers bridging the sister chromatids. These fibers are prominent in Q-positive portions. (Micrographs (a)-(c) are reproduced with permission from Ushiki et al. 2002.)

chromosomes showed that the zigzag arrangement is also closely related to the arrangement of ridges and grooves in the metaphase chromosome (Ushiki et al. 2002); the comparison of light-microscopic images with AFM findings showed that the aggregations of chromatin fibers are prominent in the regions where the chromatid is densely stained with a Giemsa solution, suggesting that the zigzag arrangement of the chromatid is produced by the presence of condensed and less condensed portions along the chromatid arm of the human metaphase chromosome. It might be also important to consider the structure of the metaphase chromosome in relation to immunocytochemical findings on the distribution of topoisomerase II $\alpha$, which is known to be a scaffold protein of the chromosome (Earnshaw \& Heck 1985, Maeshima \& Laemmli 2003, Hoshi et al. 2007a); immunofluorescence of topoisomerase II $\alpha$ is not uniform but is spotted along the chromosome axis, and the pattern of these spots in the chromosome arms seems to overlap with the banding pattern of G/Q-banded chromosomes.

We showed that a pair of sister chromatids is not completely separated even in late metaphase, but is connected by chromatin fibers especially in the ridged portions of the chromatids. Holmquist et al. (1982) considered that G-negative bands (containing GC-rich DNA) corresponded to the early clusters of 


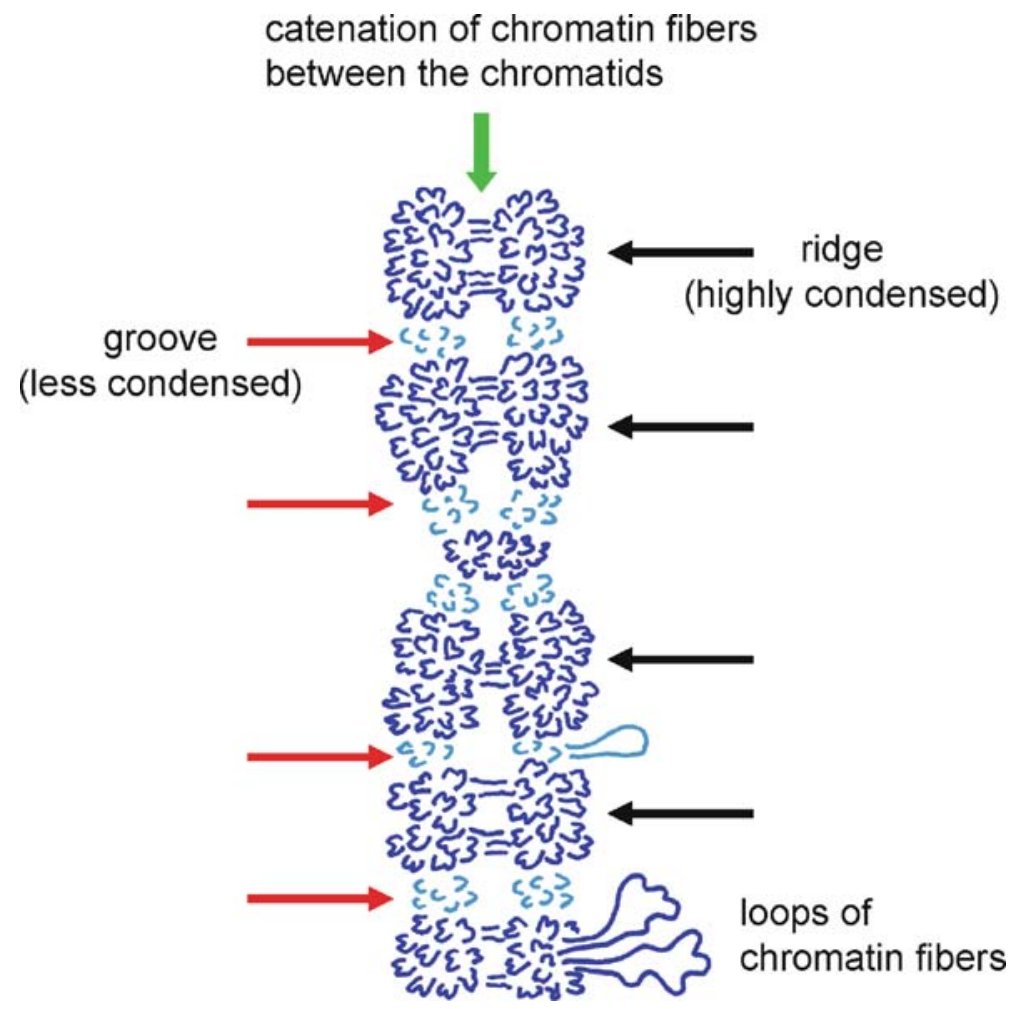

Figure 10. Schematic representation of the higher-order structure of the human metaphase chromosome. The chromosome is composed of a pair of sister chromatids with ridges and grooves. The chromatid is filled with granular and/or fibrous structures about 50-60 nm in diameter on its surface, suggesting that the chromatid is produced by the compaction of highly twisted chromatin fiber loops. The compaction of the chromatin fiber loops appears stronger in the ridged regions than in the grooved regions. The paired chromatids are often connected with entangled chromatin fibers, suggesting the presence of the catenation even in the metaphase chromosome (Hoshi et al. 2007b.)

DNA synthesis, and G-positive bands (containing AT-rich DNA) corresponded to the late clusters. Our findings might suggest that the separation of pairs of chromatids does not proceed uniformly along the chromosome arm but is delayed in the G-positive portions, resulting in the presence of catenation of chromatin fibers in regions between the ridges of the chromosome.

\section{Conclusions}

The present paper has introduced the principle of AFM as a tool for obtaining images of the threedimensional surface structure of chromosomes, and discussed our results on AFM imaging of human metaphase chromosomes for the assessment of the high-order structure of chromosomes. AFM has the characteristic of being amenable to obtaining images of samples not only in vacuum but also in air and liquid. Thus, AFM imaging of chromosomes under ambient conditions is useful for analyzing the structure of chromosomes when the sample preparations are appropriate because AFM can obtain images of the sample topography without any coating. AFM imaging of chromosomes in liquid environments is also very attractive because sample preparation can be minimized, resulting in the prevention of artifacts arising during the preparation processes hitherto employed. As a result, chromosomes can be directly observed under conditions much closer to the physiological state.

Recent advances in AFM have made it possible to collect topographic and other physical information simultaneously from the same regions of samples (Ushiki \& Kawabata, 2008). One example is the detection of local elasticity of samples together with topographic information by AFM; viscoelastic 
images obtained by this method can visualize differences in surface stiffness or local elasticity of single human chromosomes (Nomura et al. 2005; Kawabata et al. 2007). On the other hand, AFM combined with scanning near-field optical microscopy (SNOM), or SNOM/AFM is one of the candidates for an attractive tool for the study of chromosomes, because it can collect both topographic and fluorescence images of the same portion of the samples simultaneously (Iwabuchi et al. 1997; Yoshino et al. 2002, Kimura et al. 2002). Thus, SNOM/AFM is expected to be used for future studies of the structure of chromosomes in relation to the localization of their components labeled with fluorescent substances.

Furthermore, the development of high-speed AFM has advanced recently. Using this technique, topographic images of samples can be obtained at video rate (Picco et al. 2006) Thus, high-speed AFM imaging is expected to be used to investigate dynamic changes in the structure of chromosomes through direct observation of native chromosomes under liquid conditions.

\section{Acknowledgements}

This study was supported by Special Coordination Funds of the Ministry of Education, Culture, Sports, Science and Technology of the Japanese Government (to T.U.) and was supported in part by Grant-in-Aid for Scientific Research (No. 18390058) from the Japan Society for Promotion of Science, Japan (to T.U.).

\section{References}

Bak AL, Zeuthen J, Crick FH (1977) Higher-order structure of human mitotic chromosomes. Proc Nat Acad Sci U S A 74: 1595-1599.

Bath DW (1976) Surface ultrastructure of trypsin-banded chromosomes. Exp Cell Res 98: 262-268.

Bickmore WA, Craig J (1997) Chromosome Bands: Patterns in the Genome. New York: Chapman \& Hall.

Binnig G, Quate CF, Gerber C (1986) Atomic force microscope. Phys Rev Lett 56: 930-933.

Caspersson T, Zech L, Johansson C (1970) Differential banding of alkylating fluorochromes in human chromosomes. Exp Cell Res 60: $315-319$.

de Grooth BG, Putman CA (1992) High-resolution imaging of chromosome-related structures by atomic force microscopy. J Microsc 168: 239-247.

DuPraw EJ (1965) Macromolecular organization of nuclei and chromosomes: a folded fiber model based on whole-mount electron microscopy. Nature 206: 338-343.
DuPraw EJ (1970) DNA and Chromosomes. New York: Holt, Rinehart and Winston.

Earnshaw WC, Heck MMS (1985) Localization of topoisomerase II in mitotic chromosomes. J Cell Biol 100: 1716-1725.

Engel A, Lyubchenko Y, Müller DJ (1999) Atomic force microscopy: a powerful tool to observe biomolecules at work. Trends Cell Biol 9: 77-80.

Fritzsche W, Schaper A, Jovin TM (1994) Probing chromatin with scanning force microscope. Chromosoma 103: 231-236.

Fritzsche W, Vesenka J, Henderson E (1995) Scanning force microscopy of chromatin. Scanning Microsc 9: 729-739.

Fukushi D, Ushiki T (2005) The structure of C-banded human metaphase chromosomes as observed by atomic force microscopy. Arch Histol Cytol 68: 81-87.

Gall J (1963) Chromosome fibers from an interphase nucleus. Science 139: 120-121.

Harrison CJ, Britch M, Allen TD, Harris R (1981) Scanning electron microscopy of the G-banded human karyotype. Exp Cell Res 134: 141-153.

Harrison CJ, Allen TD, Harris R (1983) Scanning electron microscopy of variations in human metaphase chromosome structure revealed by Giemsa banding. Cytogenet Cell Genet 35: 21-27.

Harrison CJ, Jack EM, Allen TD, Harris R (1985) Light and scanning electron microscopy of the same human metaphase chromosomes. J Cell Sci 77: 143-153.

Holomquist G, Gray M, Porter T, Jordan J (1982) Characterization of Giemsa dark- and light-band DNA. Cell 31: 121-129.

Hörber JK, Miles MJ (2003) Scanning probe evolution in biology. Science 302: 1002-1005.

Hoshi O, Ushiki T (2001) Three-dimensional structure of G-banded human metaphase chromosomes observed by atomic force microscopy. Arch Histol Cytol 64: 475-482.

Hoshi O, Owen R, Miles M, Ushiki T (2004) Imaging of human metaphase chromosomes by atomic force microscopy in liquid. Cytogenet Genome Res 107: 28-31.

Hoshi O, Shigeno M, Ushiki T (2006) Atomic force microscopy of native human metaphase chromosomes in a liquid. Arch Histol Cytol 69: 73-78.

Hoshi O, Hirota T, Kimura E, Komatsubara N, Ushiki T (2007a) Immunocytochemistry for analyzing chromosomes. In: Fukui K, Ushiki T, eds. Chromosome Nanoscience and Technology. Boca Raton, London, New York: CRC Press, pp. 81-91.

Hoshi O, Fukushi D, Ushiki T (2007b) Atomic force microscopy of human chromosomes in relation to their high-order structure. In: Fukui K, Ushiki T, eds. Chromosome Nanoscience and Technology. Boca Raton, London, New York: CRC Press, pp. 105-117.

Inaga S, Tanaka K, Iino A (2002) Three-dimensional helical coiling structures and band patterns of hydrous metaphase chromosomes observed by low vacuum scanning electron microscopy. Arch Histol Cytol 65: 415-423.

Inaga S, Tanaka K, Ushiki T (2007) Transmission and scanning electron microscopy of mammalian metaphase chromosomes. In: Fukui K, Ushiki T, eds. Chromosome Nanoscience and Technology. Boca Raton, London, New York: CRC Press, pp. 93-104.

Iwabuchi S, Muramatsu H, Chiba N et al. (1997) Simultaneous detection of near-field topographic and fluorescence images of human chromosomes via scanning near-field optical/atomicforce microscopy (SNOAM). Nucleic Acids Res 25: 1662-1663. 
Kawabata K, Nomura K, Ikeda K et al. (2007) Mechanical approaches to elucidate mechanisms of chromosome condensation at the nano- and micro-level. In: Fukui K, Ushiki T, eds. Chromosome Nanoscience and Technology. Boca Raton, London, New York: CRC Press, pp. 1-13.

Kimura E, Hitomi J, Ushiki T (2002) Scanning neirfield optical/ atomic force microscopy of bromodeoxyuridine-incorporated human chromosome. Arch Histol Cytol 65: 435-444.

Kimura E, Hoshi O, Ushiki T (2004) Atomic force microscopy of human metaphase chromosomes after differential staining of sister chromatids. Arch Histol Cytol 67: 171-177.

Kireeva N, Lakonishok M, Kireev I, Hirano T, Belmont AS (2004) Visualization of early chromosome condensation: a hierarchical folding, axial glue model of chromosome structure. J Cell Biol 166: 775-785.

Laemmli UK, Cheng SM, Adolph KW, Paulson JA, Browns JA, Baubach WR (1978) Metaphase chromosome structure and the role of non-histone proteins. Cold Spring Harb Symp Quant Biol 42: 351-360.

Maeshima K, Laemmli UK (2003) A two-step scaffolding model for mitotic chromosome assembly. Dev Cell 4: 467-480.

Makino S (1936) The spiral structure of chromosomes in meiotic division of Podisma (Orthopetera). J Fac Sci Hokkaido Imp Univ, Ser VI 3: 29-40.

Marsden MPF, Laemmli UK (1979) Metaphase chromosome structure: evidence for a radial loop model. Cell 17: 849-868.

Meyer E, Hans JH, Roland B (2004) Scanning Probe Microscopy: The Lab on a Tip. Berlin, Heidelberg: Springer.

Musio A, Mariani T, Frediani C, Ascoli C, Sbrana I (1997) Atomic force microscope imaging of chromosome structure during G-banding treatments. Genome 40: 127-131.

Nomura K, Hoshi O, Fukushi D, Ushiki T, Haga H, Kawabata K (2005) Visualization of elasticity distribution of single human chromosomes by scanning probe microscopy. Jpn J Appl Phys 44: 5421-5424.

Ohnuki Y (1968) Structure of chromosomes. I. Morphological studies of the spiral structure of human somatic chromosomes. Chromosoma 25: 402-428.

Pease RF, Hayes TL (1966) Some biological applications of the scanning electron microscope. In: Ueda R, ed. Electron Microscopy 1966 (Proceedings of 6th International Congress on Electron Microscopy). Tokyo: Marzen, pp. 19-20.

Picco LM, Bozec L, Ulcinas A et al. (2006) Breaking the speed limit with atomic force microscopy. Nanotechnology 18: 1-4.

Putman CAJ, van er Werf KO, de Grooth BG, van Hulst NF, Greve J, Hansma PK (1992) A new imaging mode in Atomic Force Microscopy based on the error signal. SPIE 1639: 198-204.

Rattner JB (1992) Integrating chromosome structure with function. Chromosoma 101: 259-264.

Rattner JB, Lin CC (1985) Radial loops and helical coils coexist in metaphase chromosomes. Cell 42: 291-295.
Sahin FI, Rgun MAE, Tan E, Menevse A (2000) The mechanism of G-banding detected by atomic force microscopy. Scanning 22: 24-27.

Schwarzacher HG, Ruzicka F, Sperling K (1975) Electron microscopy of human banded and prematurely condensed chromosomes. Chromosomes Today 5: 227-234.

Sedat J, Manuelidis L (1978) A direct approach to the structure of eukaryotic chromosomes. Cold Spring Harb Symp Quant Bio 42: 331-350.

Sumner AT (1991) Scanning electron microscopy of mammalian chromosomes from prophase to telophase. Chromosoma 100: 410-418.

Tamayo J, Miles M, Thein A, Soothill P (1999) Selective cleaning of the cell debris in human chromosome preparations studied by scanning force microscopy. J Struct Biol 128: 200-210.

Tamayo J, Miles M (2002) Scanning probe microscopy for chromosomal research. Arch Histol Cytol 65: 369-376.

Tanaka K, Makino R, Iino A (1970) The fine structure of human somatic chromosomes studied by scanning electron microscopy and the replica method. Arch Histol Jpn 32: 203-211.

Thalhammer S, Heckl WM (2003) Atomic force microscopy in cytogenetics. In: Jena BP, Hörber JKH, eds. Force Microscopy: Application in Biology and Medicine. New Jersey: John Wiley \& Sons, pp. 249-266.

Thalhammer S, Koehler U, Stark RW, Heckl WM (2001) GTG banding pattern on human metaphase chromosomes revealed by high resolution atomic-force microscopy. J Microsc 202: 46-47.

Ushiki T (2003) Atomic force microscopy for imaging living organism: from DNA to cell motion. In: Fujita H, ed Micromachines as Tools for Nanotechnology. Berlin, Heidelberg: Springer, pp. 121-130.

Ushiki T, Kawabata K (2008) Scanning probe microscopy in biological research. In: Bhushan B, Fuchs H, Tomitori M, eds. Applied Scanning Probe Methods X. Biomimetics and Industrial Applications. Berlin, Heidelberg: Springer, pp. 285-308.

Ushiki T, Hitomi J, Ogura S, Umemoto T, Shigeno M (1996) Atomic force microscopy in histology and cytology. Arch Histol Cytol 59: 421-431.

Ushiki T, Hoshi O, Iwai K, Kimura E, Shigeno M (2002) The structure of human metaphase chromosomes: its histological perspective and new horizons by atomic force microscopy. Arch Histol Cytol 65: 377-390.

White MJD (1940) The heteropycnosis of sex chromosomes and its interpretation in term of spiral structure. $J$ Genet 40: 67-82.

Wray W, Stubblefield E (1970) A new method for the rapid isolation of chromosomes, mitotic apparatus, or nuclei from mammalian fibroblasts at near neutral pH. Exp Cell Res 59: 469-478.

Yoshino T, Sugiyama S, Hagiwara S, Ushiki T, Ohtani T (2002) Simultaneous collection of topographic and fluorescent images of barley chromosomes by scanning near-field optical/atomic force microscopy. J Electron Microsc 51: 199-203. 